\title{
Contemporaneous bilateral pallidotomy
}

\section{Robert P. Iacono, M.D., Jonathan D. Carlson, B.S.E., Sandra Kuniyoshi, B.A., Aymen Mohamed, M.D., Christine Meltzer, B.A., and Shokei Yamada, M.D.}

Departments of Neurosurgery, Physiology, and Psychology, Loma Linda University Medical Center, Loma Linda, California

To investigate the effects of bilateral posteroventral pallidotomy (PVP) for Parkinson's disease (PD), the authors reviewed the technique and neurological outcome in a number of patients who had undergone bilateral pallidotomy. The authors have previously reported the outcome of PVP in 25 bilateral and 25 unilateral surgeries, rating the patient's postural stability, speech, and total Unified Parkinson's Disease Rating Scale scores. A second, separate group of 23 patients who underwent contemporaneous bilateral PVP were evaluated for early onset idiopathic PD, levodopa failure syndrome, and severe bilateral dyskinesia as well as akinetic "off" states. Cognitive and psychological studies were reviewed in 10 patients who demonstrated preoperative impairments in spatial recognition and memory. Following surgery there were significant improvements in these cognitive deficits and no deficits were incurred by surgery.

Published reports regarding bilateral operations for PD have emphasized the risks of speech and cognitive deficits. This study shows bilateral PVP to be effective, particularly in patients with bilateral symptoms, including disabling dyskinesia. Additionally, bilateral PVP does not cause additional cognitive or speech deficits. The techniques and indications as well as outcomes and complications are presented and discussed in this report in light of their controversial nature.

Key Words * bilateral pallidotomy * speech * cognition * Parkinson's disease

Although surgical interventions for Parkinson's disease (PD) are becoming accepted for alleviating tremor, hyperkinesias, and more recently akinesia, there are still controversies concerning the safety and indications for bilateral posteroventral pallidotomy (PVP). Other considerations in these procedures include the safety of contemporaneous versus staged bilateral surgery, the most effective targeting technique, indications, complications, and long-term outcome. These issues will be discussed, with a focus on deficits and complications reported to be associated with bilateral PVP.

\section{CONTEMPORANEOUS BILATERAL PVP}

Our early experience performing PVP focused on creating unilateral or staged bilateral lesions because of the well-known hazards of creating simultaneous symmetrical deep brain lesions, particularly associated with bilateral thalamotomy.[4] We soon realized that nearly all patients with symmetric or severe appendicular symptoms were requesting a second procedure for the alleviation of persistent symptoms on 
the unoperated side. As we gained experience in the creation of a second, staged lesion, we discovered that no significant ill effects from staged bilateral lesions occurred, particularly in speech or cognitive function. We progressively shortened the time interval between the operations until we saw no further benefit in delaying the second procedure. Now a large portion of the procedures we perform are contemporaneous bilateral PVP.[15]

\section{INDICATIONS FOR CONTEMPORANEOUS PVP}

Not all patients with parkinsonian symptoms are candidates for surgery, and an even smaller subgroup are candidates for bilateral surgery. Patients with "Parkinson's plus" symptoms, such as progressive supranuclear palsy, Shy-Drager syndrome, olivopontocerebellar atrophy, and normal-pressure hydrocephalus, should not be candidates for unilateral, much less bilateral, pallidotomy because of the structural and pathophysiological differences from idiopathic PD.

We have performed surgery in a wide range of patients with idiopathic PD, ranging in age from 31 to 85 years, with severity scores measured on the Hoehn and Yahr[26] scale of 1.5/1.5 to 5.0/5.0 on and off medication. Unilateral pallidotomy alone or combined with nucleus ventralis intermedius thalamotomy is performed in patients with dominant symptoms of asymmetrical tremor.[30] Contemporaneous bilateral PVP should only be attempted in patients with severe, symmetric, bilateral, hyperkinetic appendicular disabilities including dyskinesia, who are younger than 70 years old, and who are in a psychological state such that full informed consent and cooperation can be obtained. The most common parkinsonian subgroup meeting these criteria for contemporaneous bilateral PVP are the young onset or Narabayashi's[28] so-called "juvenile" PD patient, whose age at onset is younger than 45 years old. These patients usually progress rapidly to bilateral symptoms including disabling levodopa-induced dyskinesias, as well as severe and precipitous "off" states after 5 or more years of treatment.[3,9,10,27] The medical control of the dyskinetic symptoms in these patients is not often successful because essential dopaminergic medications cause disabling dyskinesia. The decrease in dosage of these medications is usually not an option because it results in profound "off" states and severe akinetic symptoms. Bilateral PVP affords impressive elimination of all appendicular and truncal dyskinesias, dystonia, and generally precludes the development of profound "off" states, thus eliminating levodopa failure syndrome in the patients. If only a unilateral pallidotomy is performed, most of these juvenile-type patients return for surgery on the second side because of the remaining hyperkinetic symptoms, even though the majority of their quality-of-life-type parkinsonian symptoms have been alleviated.

\section{TECHNIQUE IN PVP}

Surgical techniques should be evaluated by their effectiveness, as measured by clinical outcome, reproducibility, complications, and, in this case, surgical precision. Although precise technique is essential in performing unilateral pallidotomy, an even more precise technique is required in bilateral pallidotomy to preclude neurocognitive and speech impairments. There is currently controversy about the technique for anatomical and electrophysiological target localization in the PVP. Some surgeons rely only on preoperative magnetic resonance (MR) imaging or computerized tomography (CT) scanning for anatomical targeting and use standardized coordinates measured from the anterior commissure (AC) and posterior commissure (PC); others use sophisticated stereotactic planning software as well. We have found that our preoperative targets change $40 \%$ of the time once intraoperative corrections made from third ventriculography and electrophysiological observations are accomplished. Thus, in a large percentage of cases, preoperative imaging alone does not meet the precision required for accurate 
functional lesion placement, particularly for bilateral surgery.

As published in detail elsewhere[1,7,11,13-16,19] our technique uses both preoperative target localization with MR imaging and intraoperative real-time feedback from sequential third ventriculography and orthogonal radiographs. This intraoperative anatomical target refinement integrates the MR coordinates and measurements with the landmarks of the third ventriculogram for exact probe positioning. $[14,16]$

In our experience, the most effective means for electrophysiological target finalization is the use of macroelectrode stimulation within the globus pallidus internus (GPi) of the internal capsule (at a frequency of $2 \mathrm{~Hz}$ ) and the optic tract (at a frequency of $100 \mathrm{~Hz}$ ). This macroelectrode stimulation uses an electrode with a 1.8-mm diameter and a 2-mm exposed tip (Radionics, Inc., Burlington, MA) and gives $1 \mathrm{msec}$ square wave pulses to induce synchronous threshold induction of contractures in the tongue, face, and hand through stimulation of descending internal capsular fibers at $2 \mathrm{~Hz}$. Macroelectrode stimulation at $100 \mathrm{~Hz}$ induces the appearance of stars, phosphenes, or lights in the patient's inferior contralateral visual fields by stimulating fibers in the optic tract. The minimum voltage that induces these phenomena is termed the threshold voltage. It provides an important estimate of the probe's proximity to the internal capsule and optic tract that allows for the accurate lesion placement and prevents both optic and capsule damage. Figure 1 shows a representative case of macroelectrode stimulation including the voltage thresholds for the optic tract (visual) and internal capsule (motor) as the electrode converges on these structures. The target is located in the ansa lenticularis on a course $225 \mathrm{~s}$ from the sagittal plane and 50š from the intercommissural (AC-PC) plane. Our total lesion is composed of smaller lesions that are generated as the probe is incrementally removed, with low temperatures close to the optic tract and higher temperatures above (Fig. 1). The complete lesion does not extend above the intercommissural plane. 


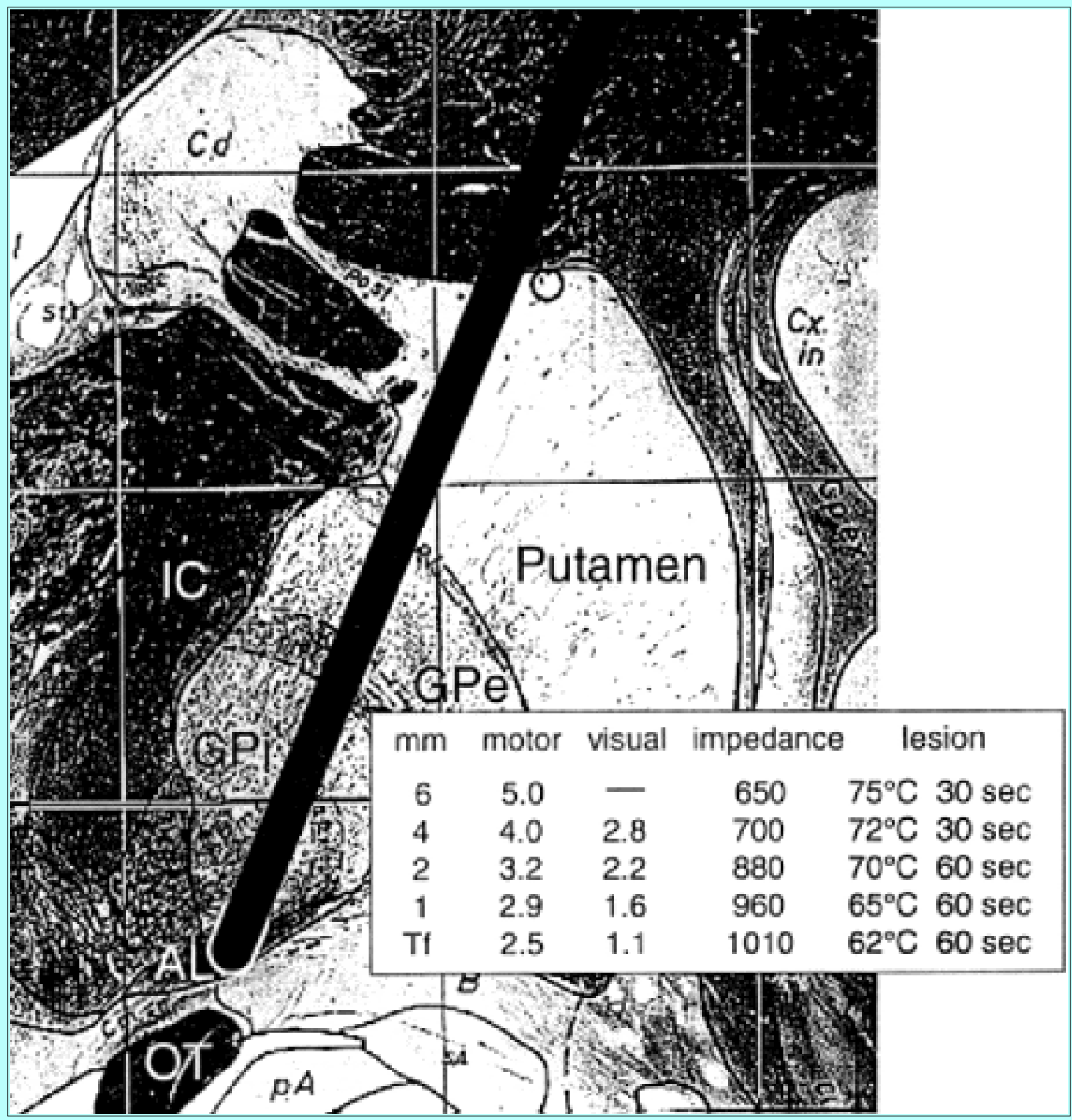

Fig. 1. Illustration of a representative case of macroelectrode stimulation for target localization with the electrode at the final target in the ansa lenticularis (AL) along a trajectory $22 \check{s}$ from the sagittal plane and 50š from the intercommissural (IC) plane.

\section{RESULTS OF CONTEMPORANEOUS BILATERAL PVP}

We present two sets of separate results, the first comparing unilateral to bilateral PVP and the second set specifically from contemporaneous bilateral PVP for early-onset idiopathic PD. We have previously reported 50 patients who underwent bilateral (25 patients) compared with unilateral (25 patients) PVPs. The patients undergoing bilateral PVP had fewer symptoms of PD following surgery and in particular experienced a decrease in axial midline symptoms, as measured by the Unified Parkinson's Disease Rating Scale (UPDRS) (Table 1).[12] Speech improved in some cases. The overall activities of daily living scores, as evidenced by the Hoehn and Yahr[26] staging scale mean scores during best "on" states were improved from 3.4 to 2.1 postoperatively. 


\begin{tabular}{|c|c|c|c|c|c|c|}
\hline \multicolumn{7}{|c|}{$\begin{array}{c}\text { TABLE } 1 \\
\text { RESULTS CONTRASTING THE EFFECTS OF UNLATERAL OR BILATERALL PVP } \\
\text { IN } 50 \text { PATIENTS }\end{array}$} \\
\hline \multirow[b]{2}{*}{ Yariatle } & \multicolumn{3}{|c|}{ Unilateral PV'P } & \multicolumn{3}{|c|}{ Bilateral PVP } \\
\hline & Preop & Postop & Diff & Preop & Postop & Diff \\
\hline $\begin{array}{l}\text { postural statility } \\
\text { speech } \\
\text { UPDRS score }\end{array}$ & $\begin{array}{l}1.5 \\
1.3 \\
53\end{array}$ & $\begin{array}{l}0.8 \\
0.7 \\
21\end{array}$ & $\begin{array}{l}0.7 \\
0.6 \\
32\end{array}$ & $\begin{array}{l}2.3 \\
1.8 \\
74\end{array}$ & $\begin{array}{l}1.0 \\
0.8 \\
25\end{array}$ & $\begin{array}{l}1.3 \\
1.0 \\
49\end{array}$ \\
\hline
\end{tabular}

In the second study we analyzed the outcome in 23 patients with early-onset idiopathic PD who underwent contemporaneous bilateral PVP. The patients showed significant improvement in their UPDRS scores, indicating the success of bilateral PVP for young onset PD, with elimination of dyskinesia (Table 2).

\begin{tabular}{|ccc|}
\hline \multicolumn{3}{|c|}{ TABLE 2 } \\
RESULTS OF PRE AND POSTOPERATIVE UPDRS SCORES IN 23 PATIENTS \\
UNDERGONG CON TEMPORANEOUS BLATERAL PVP \\
\hline \hline UPDRS Questions & ANerage "on" Preop Score & ANerage "on" Postop score \\
\hline $1-4$ & 4.06 & 0.46 \\
$5-17$ & 12.2 & 1.93 \\
$18-31$ & 15 & 2.4 \\
$32-42$ & 10.7 & 0.86 \\
total soore & 42.4 & 5.26 \\
\hline \multicolumn{3}{|c}{} \\
\hline
\end{tabular}

\section{COMPLICATION RATE}

A measure of the effectiveness of contemporaneous bilateral PVP and the accuracy of macroelectrode stimulation is the complication rate. In our recent study of 185 patients undergoing bilateral PVP, three patients experienced unilateral complications that were related to bleeding (in two) or to infection (in one). No patient undergoing contemporaneous bilateral PVP experienced bilateral complications, persistent hemiparesis, visual deficits, or significant speech or cognitive impairment. We found no significant increase in the risk of complications for patients undergoing contemporaneous bilateral PVP compared with those who underwent sequential PVP. The complications attributable to hemorrhage $(3.2 \%)$ or infection $(<1 \%)$ do not significantly exceed those associated with other series of stereotactic intracranial procedures $(2.1-4.7 \%)$. [8,20] We have not encountered other complications since we implemented surgical techniques designed to optimize localization.

\section{COGNITIVE AND PSYCHOLOGICAL STUDIES}

One remarkable effect of PVP is the statistically significant improvement in both cognitive and psychological states. The Wechsler Memory Scale[23] (WMS) with seven heterogeneous subtests was administered to 10 patients who had undergone bilateral PVP. Thirty patients received the Ray Ostereith Complex Figure Test[23] (ROCFT) and the Trail Making Test[23] (TMT). All three tests were administered 1 week before and after surgery, when the patient was in the best medicated "on" state. Moreover, we used multiple linear regression analysis to examine the relationship of reversed PD motor symptoms and improvement in cognitive functioning following PVP. Impairment in spatial recognition memory was evident on WMS, ROCFT, and TMT preoperatively in all patients. Four of five normative 
age-weighted indices for delayed recall, verbal, visual, and general memory on WMS were found to be significantly improved postoperatively $(\mathrm{p}<0.05)$. Improvement in temporal delay and frontostriatal cognitive deficits represented in WMS subscores of Logical Memory I and II, Visual Reproduction II, and Verbal Paired Associates II, showed statistically significant improvement postoperatively ( $<<0.05)$.

\section{DISCUSSION}

Bilateral surgical interventions in the basal ganglia are limited[8,16,20,21,27,30,31] and have historically been shunned because of the cognitive and speech disturbances known to often follow bilateral thalamotomy.[4] The idea that staged or sequential bilateral thalamotomy would eliminate the neurocognitive and speech effects of simultaneous bilateral thalamotomy, based on the supposition that delayed second thalamotomy should allow brain compensation through plasticity, has not been validated. Bilateral lesion position was also considered a factor in neurocognitive and speech deficits. However, even following a second small, sequential nonmirror image, lesion deficits have occurred following thalamotomy. These deficits appear to be associated with the loss of redundancy or plasticity, as might be anticipated in the atrophied aged brain or those with thalamic pathology as seen in posttraumatic tremor. Therefore, it is with some trepidation that one should consider performing bilateral ablative lesions any larger than absolutely necessary to satisfy the minimal therapeutic need.

Published reports regarding bilateral operations for PD are few. [8,16,20,21,27,30,31] Laitinen[21] reported on 12 patients who underwent bilateral pallidotomy and experienced no side effects. It seems that most of the earlier reports on bilateral pallidotomy dealt mainly with sequential bilateral procedures. Cooper[5] hesitated to sacrifice the same structure bilaterally; however, he and his coworkers[6] later published some work on simultaneous bilateral chemopallidectomy for dystonia musculorum deformans. Gillingham, et al.,[8] followed 66 cases treated by sequential bilateral pallidotomy and they pointed out that all of their patients continued to improve after the second operation. However, in one recent report, bilateral PVP performed using differing techniques has resulted in serious cognitive problems.[23]

To date we have performed contemporaneous bilateral PVP in more than 185 patients ( 370 double pallidal procedures); less than 70 have been performed sequentially. The results of this series, especially in the group of patients with advanced PD who demonstrated no lasting neurocognitive, speech, or neurological complications, strongly suggests the need for a reappraisal of bilateral interventions in the pallidum.

Based on our experience, we believe that with meticulous surgical technique, intraoperative confirmation of probe position, a cooperative patient, and proper lesion placement and size, complications can be avoided in contemporaneous bilateral PVP. Potential dangers of bilateral PVP other than speech and cognitive deficits remain, including damage to left and right corticospinal fibers at the level of the cerebral peduncle and the optic pathways.[21,22] Before we began performing simultaneous bilateral PVP, we had performed staged procedures in 64 patients with an average interval between operations of 5.3 months (range 1-14 months). Performing bilateral surgery would be counterindicated for the stereotactic neurosurgeon performing PVP in the first 50 patients and without using techniques such as we have proven reasonably safe.[1,7,16,17,19]

When bilateral pallidotomy procedures are indicated, even if the procedures will be performed sequentially, lesions should be designed and limited to be maximally therapeutic but minimally destructive. In our procedures, great care is taken to obtain one ideal lesion trajectory with small lesions confined to the infraintercommissural plane areas within the posterior GPi. 
The advantages of contemporaneous PVP are apparent, particularly in those patients with bilateral hyperkinetic symptoms: only one operation is required, which decreases the economic costs for the procedure. The patient is not subjected to the discomfort and stress of the reapplication of the head frame or the necessity of a second course of local anesthetic. More important, positional data following localization of the first PVP target can be effectively used in refining the second target, greatly improving the safety and the efficiency of targeting and creating the second lesion.

Using our technique we have found macroelectrode stimulation, which is used in every PVP procedure, to be more effective than microelectrode recording for electrophysiological localization of the optimum target. Microelectrode recording is valuable for locating the ventral boundaries of the GPi, the ansa lenticularis, the optic tract, and the internal capsule, which is important in disoriented patients who are unable to report the effect of macroelectrode stimulation of the optic tract. However mapping requires multiple trajectories, in some reports as many as 2 to $11,[2,24]$ which increases the risk of bleeding, prolongs the procedure, and potentially increases the risk of both capsular damage and infection. The ability of the macroelectrode to induce motor and visual responses at a distance from 0 to $5 \mathrm{~mm}$, indicating the position of the probe to these structures is unique because it provides real-time feedback about the probe's position relative to the site of potential lesions. Thus optimum target localization is measured by the macroelectrode stimulation thresholds.

In most cases only one trajectory is necessary (mean 1.2) because of the use of multiple modalities for target localization and refinement. Sequential lateral radiographs following ventriculography provide real-time evaluation of the probe's potential and final position with respect to the anatomical target, as indicated by the base frames sights' cross hairs. Macroelectrode stimulation, as described, provides a measure of the probe's position in relation to the internal capsule and optic tract. The low number of trajectories indicates the high degree of agreement between ventriculographic and stimulation-confirmed target. Fewer trajectories reduce the intraoperative risk, and shorten the length of the procedure to typically less than 2 hours for a bilateral PVP. The single trajectory, which entirely avoids traversing the internal capsule, in conjunction with macroelectrode target refinement allows for confinement of the lesion to the most posterior and ventral GPi and the ansa lenticularis, extending dorsally along the trajectory yet limited below the AC-PC plane. Other techniques may create larger lesions over a wider area extending dorsally or via multiple trajectories, potentially damaging pallidal projections to wide-spread frontal and thalamic areas,[18] thus creating a thalamotomy-like effect, with the concomitant potential for bilateral thalamotomy-like neurological complications. This may explain the report of serious neurocognitive complications following bilateral sequential pallidotomy by another group.[23]

Speech difficulties associated with PD are not an indication for pallidotomy because of the wide variability of outcomes, which range from increased intelligibility of speech to rare instances of worsening of hypophonia. No PD patient with relatively intact speech showed any significant worsening following bilateral PVP.

Posteroventral pallidotomy has been shown to improve significantly or alleviate all motor symptoms associated with PD including stability, gait, and posture seen in both unilateral and bilateral groups. These symptoms, which primarily involve the axial musculature, may be due to abnormalities involving the pallidal to pedunculopontine brainstem areas we have described.[1,7,16,19] Ipsilateral appendicular symptoms that are not sufficiently alleviated by unilateral pallidotomy and patients with bilateral symptomatology often require a second pallidotomy on the unoperated side to alleviate these symptoms. 
There have been misinterpretations of the results of staged bilateral pallidotomy in which patients undergoing the second pallidotomy showed a much smaller decrease in the total UPDRS score. However, evaluation of the changes in specific motor symptoms on the unoperated side shows decreases just as significant in motor symptoms following the second surgery. The somewhat modest improvements observed in those patients undergoing bilateral pallidotomy may be of significance because this group was more severely affected (Table 1); many patients had akinetic symptoms only, which are more resistant to both medical and surgical therapy.

\section{CONCLUSIONS}

The risks of bilateral surgery on the basal ganglia have been emphasized because of the speech and cognitive deficits that are incurred following staged bilateral thalamotomy. The results of this series, which include data from a significant number of patients with advanced PD indicate that no serious neurocognitive, speech, or neurological complications are sustained postoperatively; this strongly suggests the need for a reappraisal of bilateral interventions in the pallidum. In this series with limited follow up, the long-term risks cannot be determined. Minimal lesions that allow for maximum therapeutic benefit generated under vigilant observation of patient responsiveness, have not produced serious speech, cognitive, or other neurological deficits following contemporaneous bilateral PVP.

\section{References}

1. Bakay RAE: Comment on Iacono RP, Shima F, Lonser RR: The results, indications, and physiology of posteroventral pallidotomy for patients with Parkinson's disease. Neurosurgery 36:1126-1127, 1995

2. Baron MS, Vitek JL, Bakay RA, et al: Treatment of advanced Parkinson's disease by posterior GPi pallidotomy: 1-year results of a pilot study. Ann Neurol 40:355-366, 1996

3. Bostantjopoulou S, Logothetis J, Katsarou Z, et al: Clinical observations in early and late onset Parkinson's disease. Funct Neurol 6:145-149, 1991

4. Burchiel KJ: Thalamotomy for movement disorders. Neurosurg Clin North Am 6:55-71, 1995

5. Cooper IS: Parkinsonism. Its Medical and Surgical Therapy. Springfield, Ill: Charles C Thomas, 1961

6. Cooper IS, Poloukhine N, Hoen TI: Chemopallidectomy for dystonia musculorum deformans. J Am Geriatr Assoc 4:1208-1213, 1956

7. Gildenberg PL: Comment on Iacono RP, Shima F, Lonser RR: The results, indications, and physiology of posteroventral pallidotomy for patients with Parkinson's disease. Neurosurgery 36:1126, 1995

8. Gillingham FJ, Kalyanaraman S, Donaldson AA: Bilateral stereotaxic lesions in the management of parkinsonism and dyskinesias. Br Med J 2:656-659, 1964

9. Giovannini P, Piccolo I, Genitrini S, et al: Early-onset Parkinson's disease. Mov Disord 1:36-42, 1991

10. Golbe LI: Young-onset Parkinson's disease: a clinical review. Neurology 41:168-173, 1991

11. Iacono RP, Henderson JM, Lonser RR: Combined stereotactic thalamotomy and posteroventral pallidotomy for Parkinson's disease. J Image Guided Surg 1:133-140, 1995 
12. Iacono RP, Lonser RR, Kuniyoshi S: Unilateral versus bilateral simultaneous posteroventral pallidotomy in subgroups of patients with Parkinson's disease. Stereotact Funct Neurosurg 65:6-10, 1996

13. Iacono RP, Lonser RR, Oh A, et al: New pathophysiology of Parkinson's disease revealed by posteroventral pallidotomy. Neurol Res 17:178-180, 1995

14. Iacono RP, Lonser RR, Ulloth JE, et al: Postero-ventral palidotomy in Parkinson's disease. J Clin Neurosci 2:140-145, 1995

15. Iacono RP, Lonser RR, Yamada S: Contemporaneous bilateral postero-ventral pallidotomy for early onset "juvenile type" Parkinson's disease. Case report. Acta Neurochir 131:247-252, 1994

16. Iacono RP, Shima F, Lonser RR, et al: The results, indications, and physiology of posteroventral pallidotomy for patients with Parkinson's disease. Neurosurgery 36:1118-1127, 1995

17. Iacono RP, Yamada S: Posteroventral pallidotomy for patients with Parkinson's disease, in Rengachary SS, Wilkins RH (eds): Neurosurgical Operative Atlas. Park Ridge, Ill: American Association of Neurological Surgeons, 1996, Vol 5, pp 143-153

18. Jackson A, Crossman AR: Nucleus tegmenta pedunculopontinus: efferent connections with special reference to the basal ganglia, studied in the rat by anterograde and retrograde transport of horseradish peroxidase. Neuroscience 10:725-765, 1983

19. Kelly PJ: Comment on Iacono RP, Shima F, Lonser RR: The results, indications, and physiology of posteroventral pallidotomy for patients with Parkinson's disease. Neurosurgery 36:1125-1126, 1995

20. Krayenbuhl H, Wyss OAM, Yasargil MG: Bilateral thalamotomy and pallidotomy as treatment for bilateral parkinsonism. J Neurosurg 18:429-444, 1961

21. Laitinen LV: Pallidotomy for Parkinson's disease. Neurosurg Clin North Am 6:105-112, 1995

22. Laitinen LV, Bergenheim AT, Hariz MI: Leksell's posteroventral pallidotomy in the treatment of Parkinson's disease. J Neurosurg 76:53-61, 1992

23. Lezak DM: Neuropsychological Assessment, ed 2. New York: Oxford University Press, 1983

24. Lozano AM, Duff J, Trepanier L, et al: Bilateral pallidotomy: pronounced amelioration of incapacitating levodopa-induced dyskinesias but accompanying cognitive decline. Mov Disord 11:913, 1996

25. Lozano AM, Lang AE, Galvez-Jimenez N, et al: Effect of GPi pallidotomy on motor function in Parkinson's disease. Lancet 346:1383-1387, 1995

26. Martinez-Martin P: Rating scales in Parkinson's disease, in Jankovic J, Tolosa E (eds): Parkinson's Disease and Movement Disorders, ed 2. Baltimore: Williams \& Wilkins, 1993, pp281-292

27. Matsumoto K, Asano T, Baba T, et al: Long-term follow-up results of bilateral thalamotomy for parkinsonism. Appl Neurophysiol 39:257-260, 1976-1977

28. Narabayashi H: Surgical treatment in the levodopa era, in Stern GM (ed): Parkinson's Disease. 
Baltimore: Johns Hopkins Press, 1990, pp 597-646

29. Quinn N, Critchley P, Marsden CD: Young onset Parkinson's disease. Mov Disord 2:73-91, 1987

30. Svennilson E, Torvik A, Lowe R, et al: Treatment of parkinsonism by stereotactic thermolesions in the pallidal region. A clinical evaluation of 81 cases. Psychiatr Neurol Scand 35:358-377, 1960

31. Tasker RR: Simple localization for stereoencephalotomy using the "portable" central beam of the image intensifier. Confin Neurol 26:209-212, 1965

32. Tasker RR, Siqueira J, Hawrylyshyn PA, et al: What happened to VIM thalamotomy for Parkinson's disease? Appl Neurophysiol 46:68-83, 1983

Manuscript received January 28, 1997.

Accepted in final form February 24, 1997.

Address reprint requests to: Robert P. Iacono, M.D., Department of Neurosurgery, Loma Linda

University, 11234 Anderson Street, Suite 2359, Loma Linda, California 92354. email: carljon@ @sc.llu.edu 